\title{
Investigations on the Electronic Structure of Strongly Correlated Electron System Cr-Doped $\mathrm{PrFeO}_{3}$
}

\author{
Anil Kumar*1, M Kamal Warshi ${ }^{1}$, Archna Sagdeo ${ }^{2,3}$, and P. R. Sagdeo ${ }^{1 *}$ \\ ${ }^{1}$ Material Research Laboratory, Discipline of Physics, Indian Institute of Technology Indore- \\ 453552, India \\ ${ }^{2}$ Synchrotron Utilizationsection, Raja Ramanna Center for Advanced Technology, Indore- \\ 452013, India. \\ ${ }^{3}$ Homi Bhabha National Institute, Training School Complex, Anushakti Nagar, Mumbai- \\ 400094, India.
}

\section{$\underline{\text { X-ray absorption spectroscopy at Fe K-edge: }}$}

The oxidation state of $\mathrm{Fe}$ ions in $\mathrm{PrFeO}_{3}$ have been investigated by performing the XAS measurements at Fe K-edge ${ }^{1}$. Figure-S1 shows the variation of $\mathrm{x}$-ray absorption edge along with that of standard $\mathrm{Fe}_{2} \mathrm{O}_{3}$ for prepared samples. The Fe K-edge absorption data for all samples is shifted towards higher energy side from the standard $\mathrm{Fe}^{3+}$, which suggests the coexistence of mixed oxidation state of Fe. The detailed analysis of the formation of $\mathrm{Fe}^{4+}$ ions has already been discussed in the available literature ${ }^{1-3}$. It is worth noting here that the absorption edge does not shift to higher absorption energy; hence it seems that concentration of $\mathrm{Fe}^{4+}$ ions is not changing with $\mathrm{Cr}$ doping. In present investigations, since the absorption of $\mathrm{Cr} \mathrm{K}$-edge and $\mathrm{Pr}-\mathrm{L}$ edge lies at the similar energy values, therefore it's very difficult to estimate the oxidation state of $\mathrm{Cr}$ from Cr K-edge absorption. In order to extract further information, soft XAS at Fe L-edge, Cr L-edge and $\mathrm{O}$ K-edge has been carried out. Apart from this, the pre-edge feature arising around $7115 \mathrm{eV}$ arises due to quadrupolar allowed transitions and its intensity has a direct connection with the 
non-centrosymmetry around $\mathrm{FeO}_{6}$ octahedra ${ }^{4}$. The systematic decrease in the intensity of preedge feature generally suggests the increase in inversion symmetry around the transition metal atom and vice versa ${ }^{4}$. In the present case, the estimated values of Goldschmidt structural tolerance factor scales with $\mathrm{Cr}$ content, indicating the increase in the stability and tendency towards the cubic structure $\left(\mathrm{Fe}-\mathrm{O}-\mathrm{Fe}=180^{\circ}\right)$ and as a result of the same the $\mathrm{Fe}-\mathrm{O}-\mathrm{Fe}$ bond angle also increases systematically. The systematic scaling in the Fe-O-Fe bond angles or centrosymmetry might be responsible for the observed pre-edge feature with $\mathrm{Cr}$ doping.

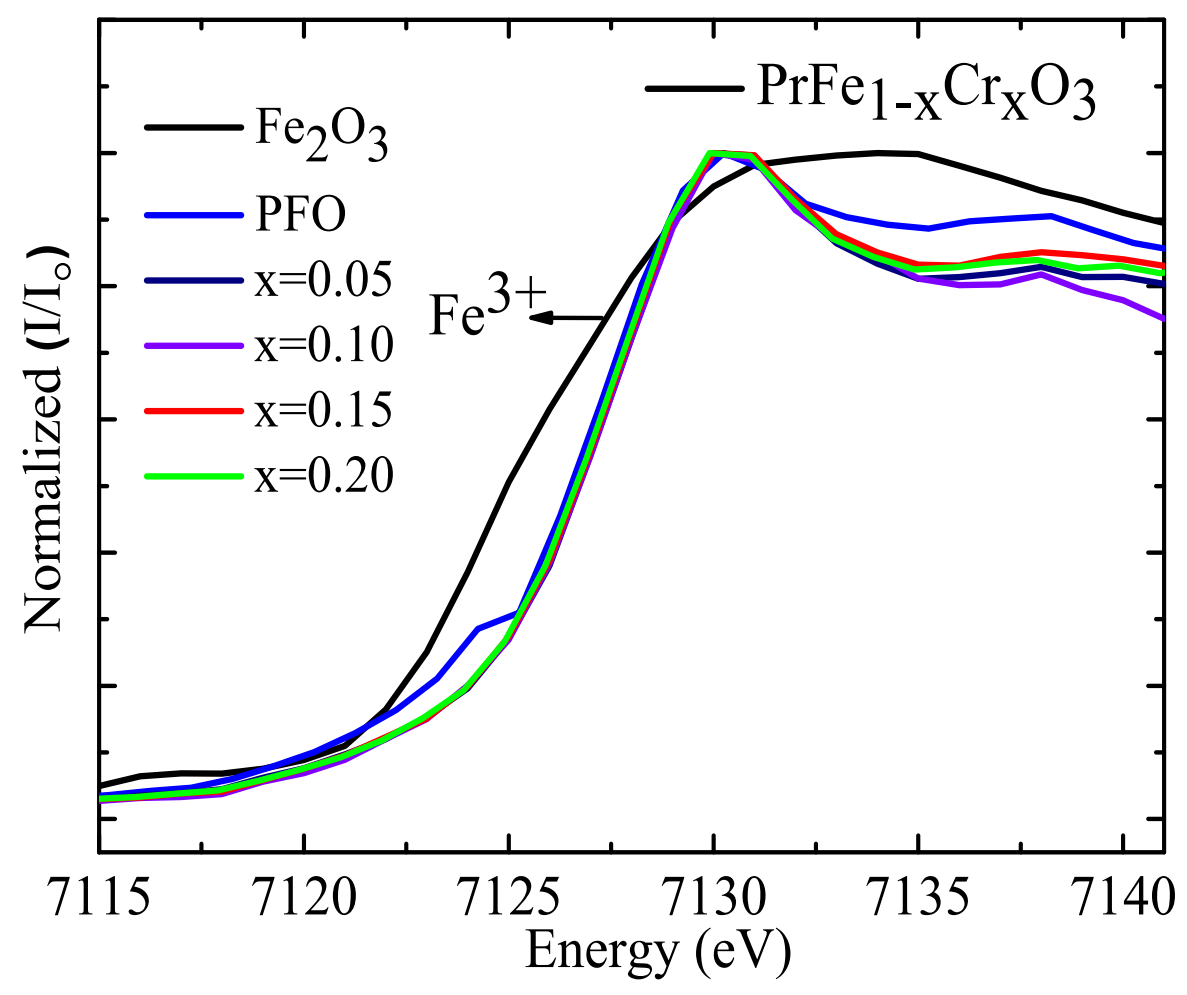

Figure-S1: The normalized Fe K-edge XAS spectra for some of the prepared series of samples. 
References:

(1) Kumar, A.; Warshi, M. K.; Sagdeo, A.; Gupta, M.; Sagdeo, P. R. New Route to Estimate the Mott-Hubbard and Charge Transfer Parameters: An Optical and x-Ray Absorption $\begin{array}{llllll}\text { Studies. } & \text { Solid } & \text { State } & \text { Sci. } & \mathbf{2 0 2 1}, & 115,\end{array}$ https://doi.org/10.1016/j.solidstatesciences.2021.106582.

(2) Kumar, A.; Sati, A.; Mishra, V.; Warshi, M. K.; Kumar, R.; Sagdeo, P. R. Charge Neutral Crystal Field Transitions: A Measure of Electron-Phonon Interaction. J. Phys. Chem. Solids 2019, 135, 109102. https://doi.org/10.1016/j.jpcs.2019.109102.

(3) Warshi, M. K.; Kumar, A.; Mishra, V.; Sati, A.; Sagdeo, A.; Kumar, R.; Sagdeo, P. R. Effect of Self-Doping on the Charge State of Fe Ions and Crystal Field Transitions in YFeO3: Experiments and Theory. J. Appl. Phys. 2019, 125 (20), 204101. https://doi.org/10.1063/1.5092736.

(4) Rai, H. M.; Saxena, S. K.; Late, R.; Mishra, V.; Rajput, P.; Sagdeo, A.; Kumar, R.; Sagdeo, P. R. Observation of Large Dielectric Permittivity and Dielectric Relaxation Phenomenon in Mn-Doped Lanthanum Gallate. RSC Adv. 2016, 6 (32), 26621-26629. https://doi.org/10.1039/C5RA28074E. 